\title{
1990 Results with Polarized Protons and Antiprotons (E704)
}

\section{(The E581/704 Collaboration)}

Past studies of spin-dependent phenomena have shown surprising effects that have not been well understood, and now new studies at Fermilab have indicated a continuation of exciting results at higher energies. An investigation of this spin dependence is important for a complete understanding of particle interactions. The remarkable results on hyperon polarization obtained at Fermilab in the 1970s sparked an interest in building a new facility that would be used to investigate a wide variety of spin-related phenomena.

The experiments E-581 and E-704 have used the Fermilab Spin Physics Facility, which consists of a $200-\mathrm{GeV}$ polarized proton or antiproton beam incident on a polarized proton target or a liquid hydrogen target, located in the meson area. This beam energy is an order of magnitude greater than for any other polarized proton beam, and the polarized antiproton beam is unique to Fermilab. The polarized beam line, which is capable of an upgrade to 500 $\mathrm{GeV}$, will be one of a kind for a long time. The construction and operation of this beam and of a polarized proton target was made possible through a significant effort by qualified staff and technical personnel at Fermilab and at the participating institutions. A substantial fraction of all experimental highenergy spin physicists from the U.S., France, Italy, Japan, and the U.S.S.R. have gathered at Fermilab to study polarization phenomena. The approved program of experiments (E-704) was intended to be of an exploratory nature, looking for new results, and was designed to precede, not follow, theoretical developments.

The polarized beam was commissioned during a short run in December 1987 and January 1988 and the design and performance are detailed in Ref. 1. At this time, the beam polarization was verified by two independent measurements ${ }^{2,3}$, and a first result on inclusive pion production ${ }^{4}$ was obtained.

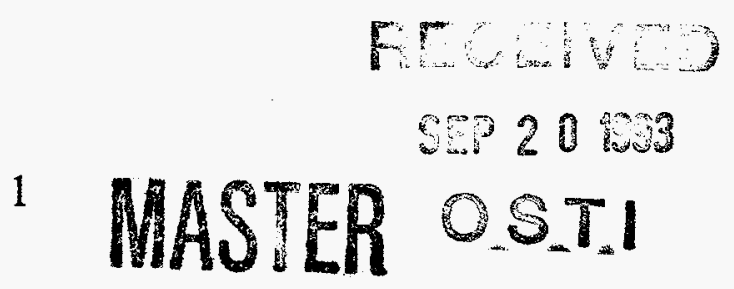




\section{DISCLAIMER}

This report was prepared as an acconnt of work sponsored by an agency of the United States Goverumeat. Neither the United States Goverment mor any agency thereof, aor any of their employeen, mikes any wrranty, equess or implied, or assumes any legal linbility or respontibility for the acomacy, compietedese, or usefuiness of any information, apparame, prodinch or process disciosed, or represents that its use would not infringe privatefy owned righte Reference hercin to any specific commercial produch, process, or service by trade mme, trodemarte manufac-

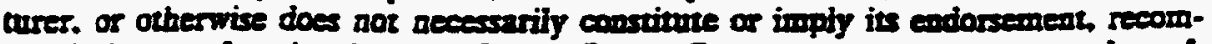
mendation. or favoring by the United Stares Goverment or any agancy thereot. The views and opinions of authors expressed berwin do not necoserrily state or refleet those of the United States Goverument or any ageosy thereof. 


\section{DISCLAIMER}

Portions of this document may be illegible in electronic image products. Images are produced from the best available original document. 
Several different spin experiments were carricd out from February to August 1990. The spin-dependent parameters, $A_{N}, A_{L L}$ and $\Delta \sigma_{L}$, measured in these experiments are defined as follows:

- The single-spin asymmetry $A_{N}$ is measured with vertically-polarized protons or antiprotons incident on a liquid hydrogen target. The parameter $A_{N}$ is the relative difference (left - right)/(left + right) of the cross sections for beam particles with spin directed vertically upward. Since the cross section for scattering or particle production to the right by beam particles with spin directed upward is the same as for production to the left by particles with spin downward, $A_{N}$ also represents the relative difference between the two cross sections for opposite beam-spin states, for production to one side of the beam.

- The two-spin asymmetry $A_{L L}$ is measured with longitudinally-polarized beam particles incident on a longitudinally-polarized proton target. The parameter $A_{L L}$ is the relative difference (antiparallel - parallel)/(antiparallel + parallel) between the cross sections for the two configurations of beam and target spins.

- $\Delta \sigma_{L}$ is the difference between the total cross sections for longitudinallypolarized beam and target particles with spins parallel or antiparallel.

When the cross sections for opposite beam spin states differ by a factor of two, the asymmmetry $A_{N}$ or $A_{L L}$, is $33 \%$, and a factor of three corresponds to an asymmetry of $50 \%$. The present Fermilab experiments have oberved large effects of this order of magnitude. This is an important result in itself since no clear prediction existed for the magnitude of the spin asymmetries that might be found at $200 \mathrm{GeV}$. The theory of strong interactions leads to fundamental spin rules governing the interactions between polarized partons, but does not predict how the partons are polarized in a polarized proton or antiproton.

Each of the different spin measurements will now be briefly described: "central" inclusive pion production, "soft" pion and $\Lambda^{\circ}$ production, total cross sections, and a new measurement of the beam polarization by the Coulombnuclear interference method. 


\section{"Central" Pion Production}

The inclusive production of neutral pions has been studied in the kinematical region where the largest possible amount of energy and momentum is exchanged in the collision between beam and target particles. The aim is to reach the region where the interaction is dominated by partonparton scattering. Neutral pions provide a good signature for the direction and energy of partons emerging from such interactions.

The single-spin parameter $A_{N}$ has been measured ${ }^{5}$, at $x_{F} \approx 0$, for the inclusive production of $\pi^{\circ}$ mesons by protons and antiprotons, and of $\eta$ mesons by protons up to the highest transverse momentum allowed by statistics. The data for $\pi^{\circ}$ production by protons have revealed unexpected features. Fig.1 shows the transition of the process from a "low- $\mathrm{p}_{t}$ " regime with zero or small asymmetry to a regime with large positive asymmetry. Experiments on inclusive pion production at lower energies had found a similar behavior. A particularly interesting observation, shown in Fig.2, is that the rise to positive values of $A_{N}$ starts for all energies at a fixed value of the transverse scaling variable $x_{t} \approx 0.4$. The quantity $x_{t}$ is the fraction of the maximal transverse momentum of the produced particle allowed by the kinematics. The Fermilab data have established that the $x_{t}$-scaling of $A_{N}$ is a high-energy phenomenon. This kinematical behavior is what one expects for the interaction of point-like objects. The lowest-order perturbative QCD amplitudes, which describe the interactions between partons, do not, however, generate any single-spin asymmetry. The result is intriguing; since it does not obey the spin rules for parton scattering, one might be tempted to conclude that the interaction is not sufficiently "hard". Very hard collisions are rare and are not yet accessible to spin experiments. However, the kinematical signature of the observed spin effect is what one would expect for parton scatttering. Possible explanations of this apparent contradiction are discussed in the literature, but the interaction dynamics responsible for the large variations of $A_{N}$ as a function of $p_{t}$ (Fig.1) are not yet understood.

This experiment has revealed that the cross sections for $\pi^{0}$ production from opposite proton transverse-spin states differ by a factor of two or more for the "hardest" collision at the highest energy attained to date in a polarization experiment. 
A measurement of the two-spin parameter $A_{L L}$ in $\pi^{\circ}$ production by protons has provided the first data for this parameter in inclusive hadronic reactions. Since the cross section differences in the interactions between partons with longitudinal spins parallel or antiparallel to each other are given by the theory of strong interactions, a measurement of $A_{L L}$ can provide information on how well the gluons or quarks "remember" the polarization of the parent proton or antiproton. The results ${ }^{6}$ for $A_{L L}$ exclude, for transverse momenta $p_{t}$ from 1 to $3 \mathrm{GeV} / \mathrm{c}$, absolute values of $A_{L L}$ larger than about $10 \%$. The statistical precision of this measurement, and therefore also the range of transverse momenta, is limited because most of the data were taken simultaneously with other measurements requiring reduced beam intensity. The result has provided an upper limit for the spin-weighted gluon distribution function in longitudinally-polarized protons. The data are in good agreement with the predictions assuming zero or small gluon polarization and do not favor the speculations that the gluon polarization might be very large. This measurement of $A_{L L}$ has not reached the $p_{t}$ region where $A_{N}$, in Fig.1, shows the significant $\mathrm{p}_{\mathrm{t}}$-dependent structure shown.

\section{"Soft" Pion and $\Lambda^{0}$ Production}

A second group of E704 measurements has studied the inclusive production of pions at relatively small transverse momentum, ranging from pions with low energy in the center of mass of the interaction to high-energy pions produced at small angles with respect to the beam direction. In this kinematical region, the momentum and energy exchanged between beam and target hadrons is small and the interactions do not reflect the scattering of individual partons. This does not mean, however, that the parton spin structure of hadrons is irrelevant to the interpretation of the observed asymmetries. In particular, for high-energy pions produced at small angles with respect to the beam, it is generally assumed that the pion is created by transfer of a leading valence quark from the beam particle.

One experiment has measured the inclusive production of charged pions. The results, given in Fig.3, show that for $x_{F} \Rightarrow 1$, protons with spin directed vertically upward produce about twice as many positive pions to the 
left as to the right, whereas negative pions are produced preferentially to the right in approximately the same ratio.

Another experiment ${ }^{7}$ has measured neutral pion production in about the same kinematical region. Fig.4 shows $A_{N}$ as a function of $x_{F}$ for neutral pions. The asymmetry increases as the pion longitudinal momentum approaches the beam momentum, in a similar manner as for charged pions.

These results for charged and neutral pions at large $x_{F}$ have established the existence of a strong spin dependence in "soft" pion production at the highest energy presently accessible. One possible interpretation attributes the observed spin dependence to the process where a leading u-quark, for $\pi^{0}$ and $\pi^{+}$production, or a d-quark for $\pi^{-s}$, is transferred from the beam proton to the produced pion. The absolute value of the asymmetry increases because the transfer process becomes dominant as $x_{F} \Rightarrow 1$. This dynamical picture assumes that the leading valence quarks in transversely polarized protons are also polarized in the transverse direction. The reason for opposite signs in $\pi^{+}$and $\pi^{-}$production could be that the leading $u$ and $d$ quarks are polarized in opposite directions. An alternate explanation proposes that the observed single-spin asymmetries are not due to the polarization of the valence quarks, but to the orbital angular momentum of the constituents in a spinning proton.

The asymmetry parameter $A_{N}$ in charged and neutral pion production has also been measured with incident antiprotons. The results, in Fig.5, for $\pi^{0}$ production by antiprotons ${ }^{8}$ show that $A_{N}(\bar{P} P)$ has the same sign and a similar $x_{F}$ dependence as $A_{N}(P P)$, but that the magnitude of $A_{N}(\overline{P P})$ is smaller. The two reactions are related by charge conjugation of the beam particle and of the produced particle, but not of the target. The observed difference of the asymmetries at large $x_{F}$ implies that the interactions inyolve constituents in the target proton that are not invariant under charge conjugation.

In addition to the spin asymmetries, the ratio $R(\tilde{P} / \mathrm{P})$ of the spinaveraged cross sections has been determined from the data. The results ${ }^{8}$ show a strong dependence on the kinematics, with values ranging from $R=1$ to $R=$ 2. The statistical accuracy here is substantially better than in previous measurements using unpolarized beams that contain fewer antiprotons. 
The analysis of the data for charged-pion production by antiprotons, which is still in progress, will complete the set of E704 measurements for pion production at $\mathrm{p}_{\mathrm{t}}<2 \mathrm{GeV} / \mathrm{c}$. This first set of high-energy data provides a large amount of input and significant constraints for the phenomenology of "soft" pion production by protons and antiprotons in definite transverse-spin states.

The data analysis for $\Lambda$ hyperons produced in the same kinematical region is also still in progress, with an expected total of about $1.5 \times 10^{5}$ reconstructed events. This experiment measures the spin dependence of the production cross sections and the correlation between the transverse beam polarization and the polarization of the produced particle. The results will be compared with the expectations from models constructed to explain why $\Lambda \mathrm{s}$ are polarized when produced with unpolarized beam and target.

\section{Total Cross Sections}

Using a polarized beam and a polarized target, one can determine if the interactions with parallel and antiparallel beam and target spins contribute equally or differently to the spin-averaged total cross section. The cross section measurement was made with high statistical precision for both incident protons and antiprotons. A preliminary analysis shows that the difference $\Delta \sigma_{\mathrm{L}}$ is small for either reaction. Some models, which successfully relate differences in cross sections among various particles to counting of valence quarks, predict that $\Delta \sigma_{L}$ for antiprotons should differ from that of protons by about a millibarn. Our ultimate experimental errors should be an order of magnitude smaller than this prediction.

\section{Coulomb-Nuclear Interference}

Interactions used at lower energies to measure beam polarizations could not be applied at Fermilab energies because either the analyzing power or the counting rates would have been prohibitively low. Two new methods were introduced in 1987-88, one based on the Primakoff effect, i.e., nucleon-isobar production by interaction of the beam particle with the electromagnetic field of a heavy nuclear target ${ }^{2}$, and the other based on the interference between 
Coulomb and nuclear scattering ${ }^{3}$ in proton-proton clastic scattering at small angles. The second measurement was repeated, with improved experimental conditions, during the E704 run in 1990. The new results have confirmed the proton beam polarization with three times better precision. Also, the dependence of the asymmetry on the four-momentum transfer, $-t$, has been measured for the first time as a verification of the method.

\section{Su m m ary}

The results from the highest-energy polarized proton and antiproton beam, available as of January 1991, can be summarized as follows:

* The single-spin results for $\pi^{0}$ production at $x_{F} \approx 0$ and $x_{t} \approx 0.4$ are important not only because the spin effect is large and exhibits $x_{t}$-scaling, but also because transverse-spin effects in general present a particular challenge for the theoretical interpretation. This contrasts with longitudinal spin effects which can be related to parton helicities.

* The measurements of neutral and charged pion production by protons and antiprotons at pion transverse momenta of $\mathrm{p}_{t} \leq 2 \mathrm{GeV} / \mathrm{c}$ represent an extensive set of high-energy data that will provide a complete, clear picture of the experimental situation in "soft" pion production at $200 \mathrm{GeV}$.

* The measurement of the two-spin parameter $A_{L L}$ has not reached the statistical precision and the highest $\mathrm{p}_{\mathrm{t}}$-values attainable at Fermilab. This parameter is of particular interest because, in hadronic reactions that are dominated by parton-parton scattering, $A_{L L}$ is related to the spin properties of the parton scattering amplitudes and to the spin-weighted parton helicity distributions.

Work supported in part by the U. S. Department of Energy, Division of High Energy Physics, Contract W-31-109-ENG-38. 
D.L.Adams(19), N.Akchurin(6), N.I.Belikov(5), B.E.Bonner(19), A.Bravar(20), J.A.Buchanan (19), J.Bystricky(2), J.M.Clement(19), P.Chaumette(2), M.D.Corcoran(19), J.D.Cossairt(3), J.Cranshaw(19), J.Deregel(2), A.A.Derevschikov(5), G.Durand(2), H.En'yo(8), J.Fabre(2), K.Fukuda(13), H.Funahashi(8), Y.Goto(8), O.A.Grachov(5), D.P.Grosnick(1), D.A.Hill(1), K.Imai(8), Y.Itow ${ }^{(8)}$, K.Iwatani $(4)$, T.Kasprzyk $(1)$ K.W.Krueger(15), K.Kuroda(11), M.Laghai(1), J.Langland(6), F.Lehar(2), A. de Lesquen(2), D.Lopiano(1), F.C.Luehring(16)(a), T.Maki(7), S.Makino(8), A.Masaike(8), Yu.A.Matulenko(5), A.P.Meschanin(5), A.Michalowicz(11), D.H.Miller(16), K.Miyake(8), A.I.Mysnik(5), T.Nagamine(8)(b), F.Nessi-Tedaldi(19)(c), M.Nessi(19)(c), C.Nguyen (19), S.B.Nurushev(5), Y.Ohashi(1), Y.Onel(6), D.I.Patalakha(5), G.Pauletta(21), A.Penzo(20), G.C.Phillips(19)(d), A.L.Read(3), J.B.Roberts(19), L.van Rossum(1)(3), V.L.Rykov(5), R.A.Rzaev(5), N.Saito(8), G.Salvato(14), P.Schiavon(20), P.N.Shanahan (16), J.Shepard(1), J.Skeens(19), L.F.Soloviev(5), V.L.Solovyanov (5), H.Spinka(1), R.W.Stanek ${ }^{(1)}$, R.Takashima ${ }^{(9)}$, F.Takeutchi(10), N.Tamura(17), N.Tanaka(12), D.G.Underwood(1), A.N.Vasiliev(5), A.Villari(14), J.L.White(19), S.Yamashita(8), A.Yokosawa(1), T.Yoshida(18), A.Zanetti(20).

(1) Argonne National Laboratory, Argonne, Illinois 60439,USA;

(2) CEN-Saclay, F-91191 Gif-sur-Yvette, France;

(3) Fermi National Accelerator Laboratory, Batavia, Illinois 60510,USA;

(4) Hiroshima University, Higashi-Hiroshima 724, Japan;

(5) Institute of High Energy Physics, Serpukhov, USSR;

(6) Department of Physics, University of Iowa, Iowa City,Iowa 52242,USA;

(7) University of Occupational and Environmental Health, Kita-Kyushu

(8) Department of Physics, Kyoto University, Kyoto 606, Japan;

(9) Kyoto University of Education, Kyoto 612, Japan;

(10) Kyoto-Sangyo University, Kyoto 612, Japan;

(11) Lab. de Physique des Particules, BP 909, F-74017 Annecy-le-Vieux, France;

(12) Los Alamos National Laboratory, Los Alamos, New Mexico 87545, USA;

(13) Meiji College of Oriental Medicine, Funai-gun, Kyoto-fu 629-03, Japan.

(14) Dipartimento di Fisica,University of Messina,I-98100, Messina, Italy;

(15) Northeastern State University, Talequah, Oklahoma 74464,USA ;

(16) Physics Department, Northwestern University, Evanston, Illinois 60201,USA;

(17) Okayama University, Okayama 700, Japan;

(18) Osaka City University, Osaka 558, Japan;

(19) T.W.Bonner Nuclear Laboratory, Rice University, Houston, Texas 77251,USA; (20) Dipartimento di Fisica, University of Trieste, I-34100, Trieste,Italy;

(21) University of Udine, 33100 Udine, UD,Italy.

Present address:

(a) Indiana University, Bloomington, Indiana 47405, USA

(b) Stanford Linear Accelerator Center, Stanford, California 94305, USA

(c) CERN, Ch-1211 Geneva 23, Switzerland

(d) Columbia Scientific Industries, Austin, Texas 78710,USA 


\section{REFERENCES}

For references on the theoretical interpretation of $A_{N}$ and $A_{L L}$, and on related experiments at lower energies, see the E704 publications 4. to 8.

1. D.P.Grosnick et al., Nucl.Instrum.Meth.A290,269(1990).

2. D.C.Carey et al., Phys.Rev.Lett.64,357(1990).

3. N.Akchurin et al., Phys.Lett.B229,299(1989).

4. B.E.Bonner et al., Phys.Rev.Lett.61,1918(1988)

5. D.L.Adams et al., Preprint FERMILAB-Pub-91/14-E,January 1991, submitted to Phys.Rev.Lett.

6. D.L.Adams et al., Preprint FERMILAB-Pub-91/16-E,January 1991, submitted to Phys.Lett.

7. D.L.Adams et al., Preprint FERMILAB-Pub-91/13-E,January 1991, submitted to Phys.Rev.Lett.

8. D.L.Adams et al,, Preprint FERMILAB-Pub-91/15-E,January 1991, submitted to Phys.Lett. 


\section{FIGURE CAPTIONS}

FIG.1 The asymmetry parameter $A_{N}$ in the reaction $P+P \Rightarrow \pi^{\circ}+X$ at $200 \mathrm{GeV}$ as a function of $\mathrm{p}_{\mathrm{t}}$ at $\mathrm{x}_{\mathrm{F}} \approx 0$. The dotted line is added to guide the eye. $\sigma \uparrow / \sigma \downarrow$ is the ratio of the $\pi^{\circ}$ production cross scctions for opposite beam spins.

FIG.2 The onset of the rise of $A_{N}$ to large positive values (closed circles) for different C.M.energies, showing data from this experiment and from the previous experiments at lower energies.

FIG.3 Preliminary results for the asymmetry parameter $A_{N}$ in the reactions $P+P \Rightarrow \pi^{+}+X$ (full squares) and $P+P=>\pi^{-}+X$ (open circles) at $200 \mathrm{GeV}$, as a function of $x_{F}$, integrated over $p_{t}$.

FIG.4 The asymmetry parameter $A_{N}$ in the reaction $P+P \Rightarrow \pi^{\circ}+X$ at $200 \mathrm{GeV}$ as a function of $x_{F}$, integrated over $0.5<\mathrm{p}_{\mathrm{t}}<2 \mathrm{GeV} / \mathrm{c} . \sigma \uparrow / \sigma \downarrow$ is the ratio of the $\pi^{\circ}$ production cross sections for opposite beam spins.

FIG.5 The asymmetry parameter $A_{N}$ in the reaction $\bar{P}+P=>\pi^{0}+X$ (full circles) at $200 \mathrm{GeV}$ as a function of $\mathrm{x}_{F}$, integrated over $0.5<\mathrm{p}_{\mathrm{t}}<2 \mathrm{GeV} / \mathrm{c}$. $\sigma \uparrow / \sigma \downarrow$ is the ratio of the $\pi^{\circ}$ production cross sections for opposite beam spins. The corresponding data for the reaction $P+P \Rightarrow \pi^{0}+X$ are shown as open squares. 


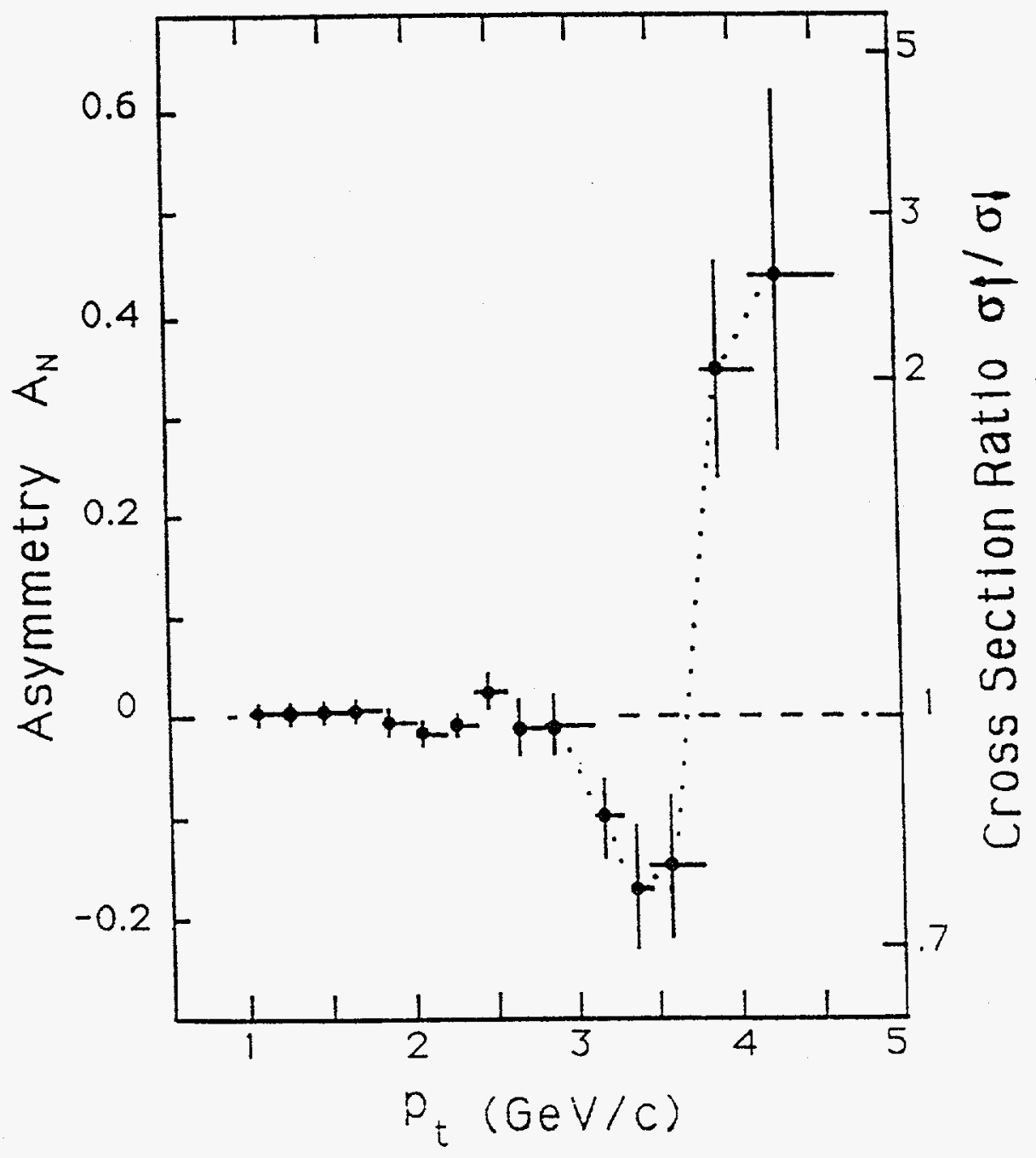

Figure 1 


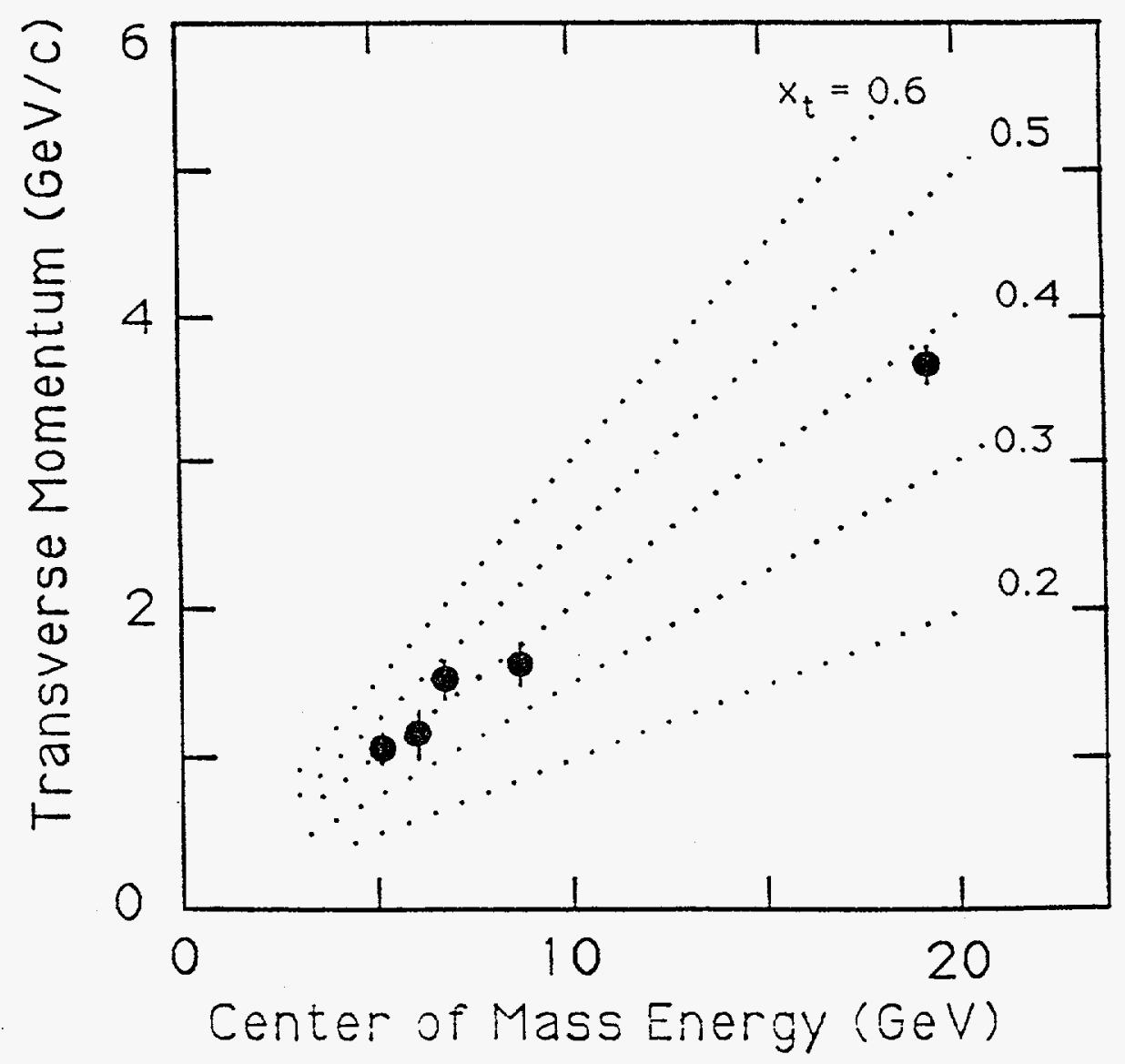

Figure 2 


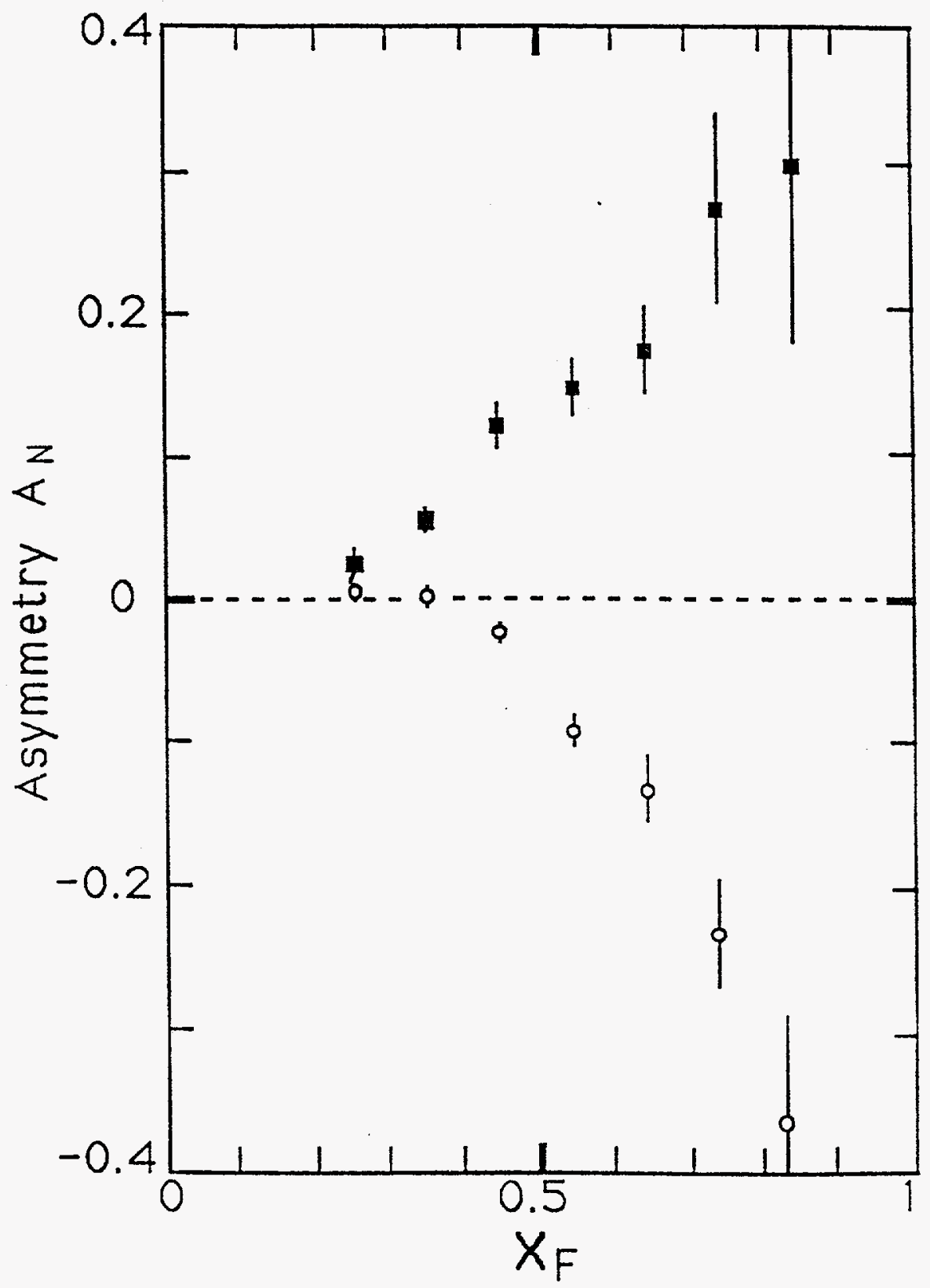

Figure 3 


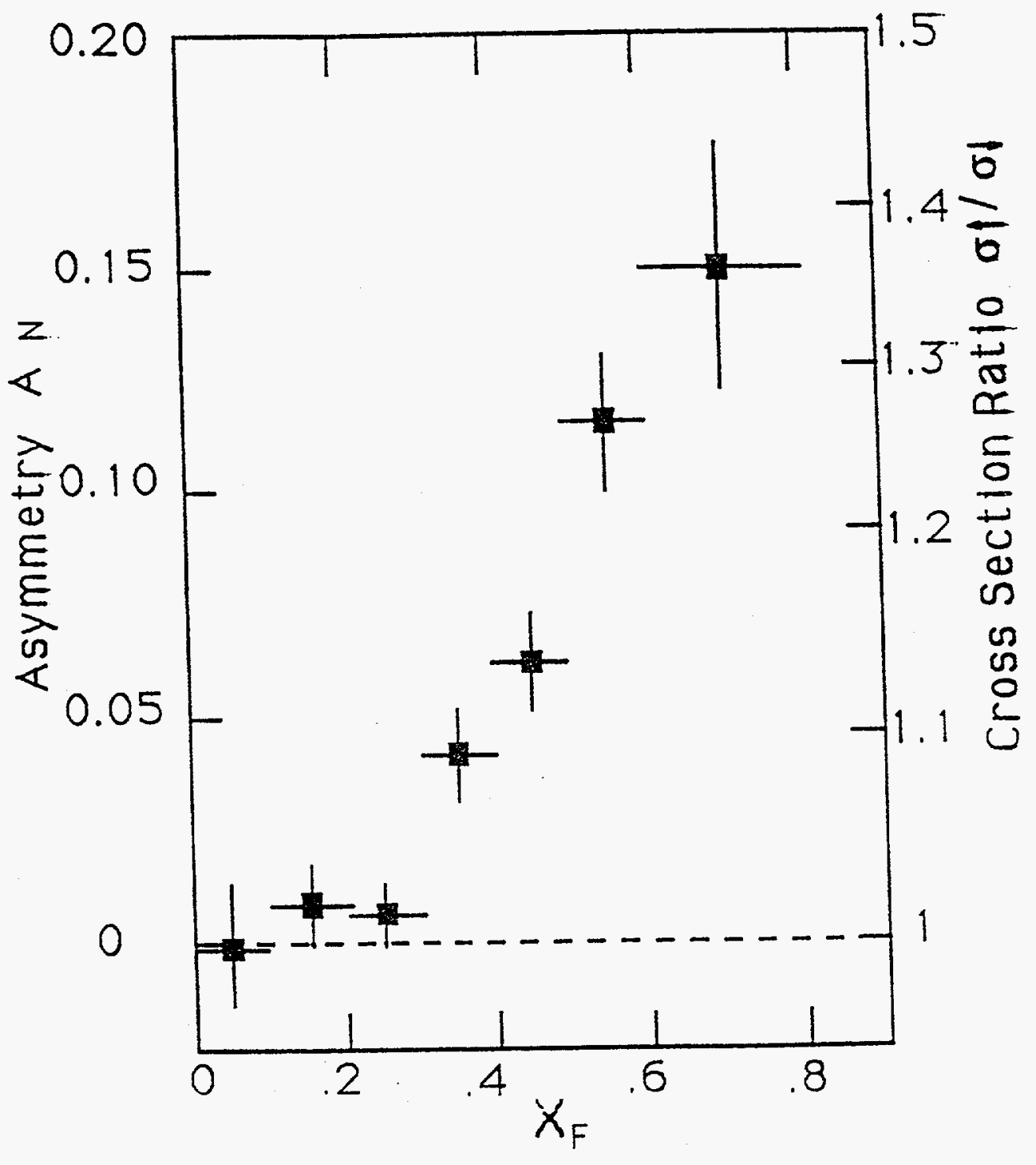

Figure 4 


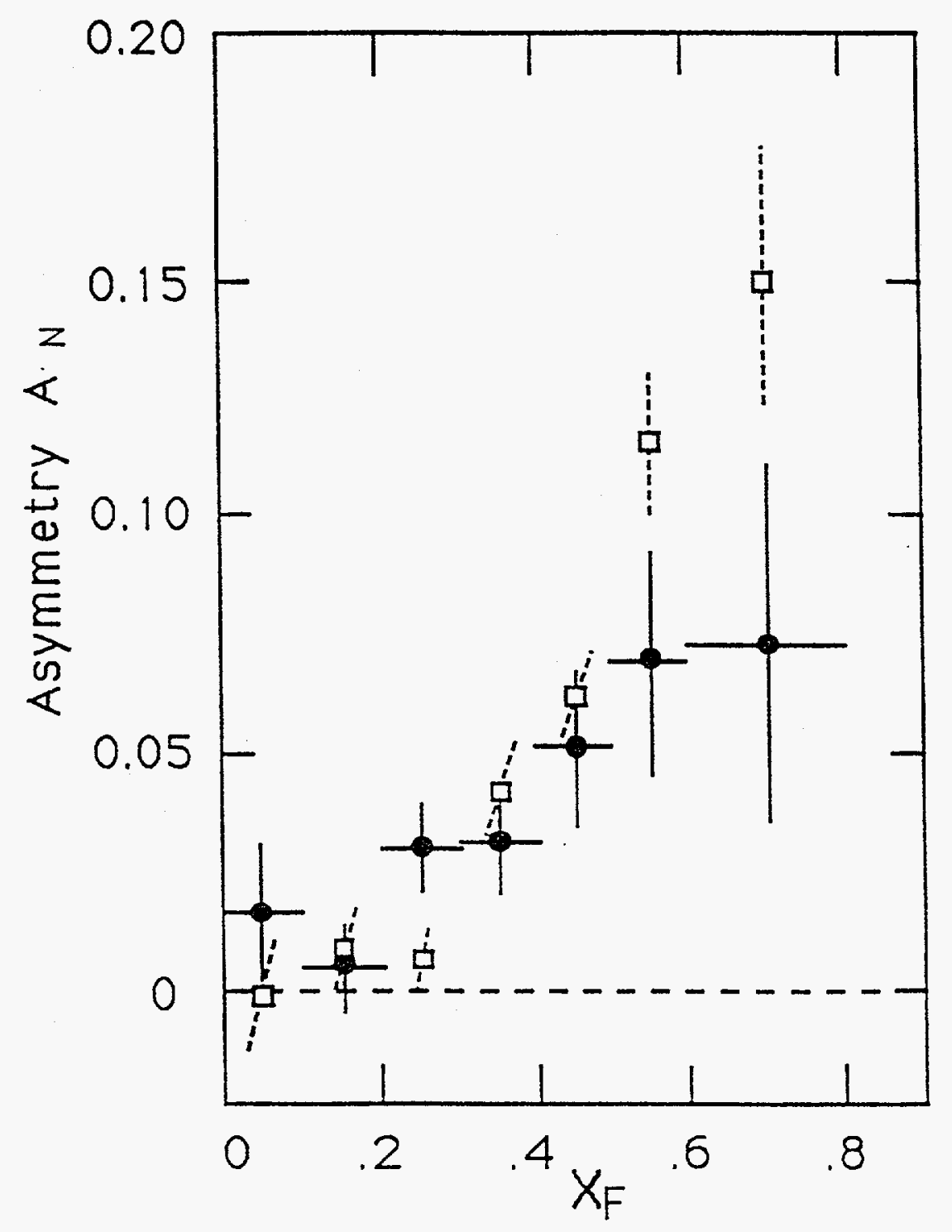

Figure 5 